\title{
Building a University-Wide Agenda for Intercultural Competence and Understanding: Lessons Learned at the University of Minnesota
}

\author{
Andrew Furco and Kristin Nering Lockhart
}

\begin{abstract}
Furthering intercultural competence in higher education requires colleges and universities to establish an intentional, mission driven strategic plan that embeds intercultural understanding and practice across the institution's work. To secure broad-based buy-in and support, this plan needs to consider the various ways that different units within the academy define, interpret, and view intercultural work. For large, complex and multi-faceted colleges and universities, building this plan can prove daunting as different parts of the institution will ascribe different meanings, purposes, and intentions to intercultural advancement.

In this paper, administrators from the University of Minnesota, who represent units that are highly engaged in intercultural competence work, share some of their struggles and lessons learned in their effort to build a comprehensive, campus-wide strategy to grow intercultural advancement. The administrators initiated this campus-wide strategic work following their participation in an international conference focused on intercultural competence. Since that time, they have developed recommendations for the foundational work required to establish a common university-wide framework and comprehensive plan for institutional intercultural advancement.
\end{abstract}

Keywords: public engagement; equity; diversity; inclusion

\section{Inspiration and Good Intentions}

With one of us serving as an associate vice president responsible for addressing issues of equity and diversity across the institution, and the other serving as an associate vice president responsible for advancing the institution's community and public engagement agenda, discussions about the importance of intercultural competence have long been familiar to us. For the Associate Vice President for Equity and Diversity, having inter-culturally competent faculty, staff, and student body is essential for securing a culturally sensitive and welcoming climate for our University's diverse, multicultural community. For the Associate Vice President for Public Engagement, having inter-culturally competent faculty, staff, and students is essential to ensure effective, high quality practice when engaging the University in partnerships with diverse external community constituents. While the issue of ensuring high intercultural competence among our respective stakeholders has remained central for both our offices, we found ourselves engaged in few, if any, focused cross-unit discussions or collaborations on the subject.

In 2011, we serendipitously learned about an international conference focused on exploring the intersection of community service-learning and intercultural development in diverse settings, and we began to consider how we, as senior university administrators, might use the event to join forces and develop a university-wide agenda for advancing intercultural competence and

Metropolitan Universities Vol. 29 No. 1 (February 2018), DOI: 10.18060/22171 
understanding. Our belief was that such as agenda would bring greater attention to and demonstrate the importance of securing intercultural understanding among our faculty, staff, and students.

Set in the idyllic city of Siena, Italy, this international conference, hosted by Siena Italian Studies and International Horizons, ultimately provided a space for us to examine intercultural competence through a fresh perspective. While the conference focused on exploring intercultural issues in service-learning and university-community partnership work, it brought to the fore the importance of an intentional, strategic agenda focused on securing intercultural competence campus-wide. Intercultural competence does not manifest automatically within college campuses. It needs to be attended to through carefully crafted professional development opportunities (Deardorff, 2009). As we learned at the Siena conference, intercultural competence cannot be achieved unless there is an institutional culture and intentional action that genuinely support its cultivation and development.

Participation in the conference provided us the opportunity not only to articulate, reflect on, and explore our respective perspectives on intercultural understanding, but it enlightened us on how our respective perspectives, while similar in some respects, were quite distinct. The public engagement-focused work, for example, emphasizes issues of developing the intercultural competence of students, faculty, and staff so that they can successfully navigate the cultural, ethnic, and disciplinary obstacles to the advancement of effective community-partnered work. In this regard, having intercultural competence is important for ensuring that partnership transaction is conducted with quality (e.g., high impact work that has mutual benefits).

In contrast, the equity and diversity work focuses on building a community within the University that embraces, nurtures, and takes advantage of the lived experiences, talents, and world views of those holding diverse, and often multiple, identities. Here, intercultural competence is important to establish a campus environment that allows the individuals who research, educate and work within it to belong and to thrive. While the externally focused public engagement perspective and the internally focused equity and diversity perspective differed in their emphases and orientations, both spoke to the University's core value that excellence is achievable only in an environment that fully supports engagement with those from diverse cultures and with differently informed perspectives.

As we began to learn more about each other's approach to intercultural issues, it became clear that to build a University-wide agenda focused on building intercultural competence, we needed to deepen understanding of the nuances of intercultural work within each of our function areas. In addition, our presence at the Siena conference also exposed us to unique intercultural issues that are prevalent in international service-learning experiences. With internationalization as another strategic goal of our institution, we brought in the University's associate vice president for international programs to join our effort.

By the end of the conference, we agreed to form an intercultural development groupIntercultural Competence Work Group - that would bring together the various units and key stakeholders from across the institution for whom intercultural competence and understanding are essential to their work. After a period of planning and development, we invited and gathered 
colleagues from across the institution to explore interest in and the viability of building a University-wide agenda for advancing intercultural understanding.

\section{Toward a Collective Agenda}

From the start of our journey, it was clear that structure, history, size, culture, and politics of our institution, the University of Minnesota, all influenced the ways in which intercultural work is operationalized within units. As with any institutional change initiative, embracing the essence of the institution, its identity and sense of what it wants to be, would be key to building an institution-wide agenda for intercultural understanding. The first set of phases of the group's work can be characterized as the discovery and unpacking phases during which we spent much time in exploring the definitions and diverse purposes of intercultural work, and the shared goal of creating an institution-wide agenda for supporting the system-wide growth of intercultural understanding.

The University of Minnesota is a multi-faceted public, land-grant research University in the midwestern part of the United States. It enrolls more than 55,000 students across five campuses that each have unique strengths, missions and identities. To establish an institution-wide agenda requires the genuine and full involvement of key administrative and academic units engaged in intercultural work across the University. In order to be sustainable, intercultural advancement could not be the work of only some units or programs, but rather needed be part of everyone's work. Therefore, while the various intercultural work being done across our institution was rich and often rooted in long standing deep partnerships, we were sure that with an intentional, strategic, and collective effort, we could leverage greater impact from all of the resources invested in these efforts.

The group was led by three associate vice presidents (public engagement, equity and diversity, and international programs) responsible for managing University-wide senior-level offices for which intercultural work is central. We convened representatives from key units from across the system who we felt were engaged in intercultural work, beginning with administrators involved in public engagement, equity and diversity, global programs and student affairs. ${ }^{1}$ There was strong consensus among group members that the institution was in need of a University-wide agenda focused on building an institutional culture that saw intercultural understanding as central to the University's work. In fact, there was support from the group and great excitement at the prospect of having an intercultural advancement agenda in place.

\section{Phase I: Reconciling Differences}

It did not take long for us to realize that the path to our intended outcomes would be more complicated, require more effort, and take far more time than any of us anticipated. We learned very quickly that the participating units did not operationalize intercultural work in the same way or viewed intercultural competence through the same lens. We spent the first part of our group's work learning about the nature and extent of each other's intercultural work, with the shared purpose of finding a way to build a University-wide agenda to advance intercultural

\footnotetext{
${ }^{1}$ Over time, other members of the university community from administrative and collegiate units asked to be included in this work, and were considered critical partners in this effort.
} 
understanding. As we considered how best to go about engaging the various units and developing with a cohesive, common agenda for intercultural advancement, we identified the specific intercultural-related needs and challenges that we faced at the University and within our respective units. It was during this exploration that the process and prospect of establishing a University-wide agenda began to unravel as the cultural, structural, and historical contexts of the University strongly influenced the ways in which inter-culturalism was perceived and operationalized in different units.

During this initial phase, five steps proved important in reconciling the differences among members' perspectives and viewpoints on the subject: (1) defining terms and concepts; (2) agreeing on a new term to define the group's work; (3) building greater cross-unit alignment; (4) securing administrative support; and (5) questioning the validity and value of standardized assessments used in intercultural work.

\section{Defining Terms and Concepts}

As each unit representative shared the different frames, lenses, ways of thinking regarding his/her intercultural work, we grappled with the question: "How can we build an institution-wide agenda that leverages our common goals without compromising or devaluing our individual frames and perspectives on intercultural work?” For some time, our group struggled mightily to hone the definition of "intercultural competence". What exactly did we mean by this term? Our discussions focused not only on the term 'intercultural', but also on whether 'competence' was even achievable, or whether intercultural competence should even be set as an aspirational goal. Indeed, the definitional issues surrounding the meanings, purposes, and focuses of intercultural competence presented the greatest challenges to our group's work, causing us to substantially shift directions as the work proceeded. It should be noted that, in hindsight, it was the work of unpacking the conceptual messiness of the different interpretations of intercultural competence that ultimately strengthened the group's commitment to establishing a campus-wide agenda and to building stronger relationships across administrative units.

Through a series of robust discussions, which included the engagement of the group's members in a half-day retreat, five primary frames for intercultural development emerged (See Table 1). Each area, while focused on a different set of goals, promotes the development of intercultural competence.

Table 1. Intercultural Competence Development Frames

\begin{tabular}{|l|l|}
\hline Frame & Focus \\
\hline Intercultural Communication & $\begin{array}{l}\text { Understanding how people from different countries and } \\
\text { cultures act, communicate, and perceive the world around } \\
\text { them. }\end{array}$ \\
\hline Equity and Diversity & $\begin{array}{l}\text { Securing equal opportunities, access, and representation } \\
\text { within and across identified cultural, social, and religious } \\
\text { groups. }\end{array}$ \\
\hline Social Justice & $\begin{array}{l}\text { Securing the fair and proper administration of laws and the } \\
\text { equal treatment without prejudice to all persons, }\end{array}$ \\
\hline
\end{tabular}




\begin{tabular}{|l|l|}
\hline & $\begin{array}{l}\text { irrespective of ethnic origin, gender, possessions, race, } \\
\text { religion, ability, sexual orientation, status, etc. }\end{array}$ \\
\hline $\begin{array}{l}\text { Social Change- } \\
\text { Leadership Development }\end{array}$ & $\begin{array}{l}\text { Preparing leaders as change agents for society's most } \\
\text { challenging issues. }\end{array}$ \\
\hline Cultural Interfacing & $\begin{array}{l}\text { Interacting with (rather than reading about) new and } \\
\text { different cultures in meaningful ways that promote one's } \\
\text { deep understanding of cultural norms and nuances. }\end{array}$ \\
\hline
\end{tabular}

One or more of these development frames resonate with, and are a central part of the intercultural work within, the three overarching, organizing units of our agenda-setting group equity and diversity, public engagement, and international programs.

Agreeing on a New Term

Given that each member of the group had preconceived views on what intercultural competence entailed, it became clear to us that the term itself was problematic, since when used, it would anchor the group members' thinking to the particular perspectives with which they were most familiar. We encouraged group members to model intercultural understanding by asking them to work to better understand what was at the core of other members' intercultural competence perspectives and frames. To do this, we dispensed with the term "intercultural competence" and agreed to move forward to develop an agenda for a new construct that would be named at a later time.

\section{Building Greater Cross-Unit Alignment}

Within the units represented in the work group, there was much activity underway focused on enhancing intercultural understanding. This suggested that the University was making noteworthy and significant investments to support the issue. Nevertheless, the group agreed that despite robust unit-level efforts, the University lacked cross-unit collaborations that could be used to reinforce mutual goals and promote greater impact through collective action. The group also agreed that the institution needed to bring greater awareness and visibility of our group's work, demonstrate a more visible institutional commitment to the issue, and provide more widespread support for moving the agenda forward. While the group acknowledged that the decentralized structure and culture of the institution challenged our capacity to build a coordinated and unified agenda, it saw much potential for achieving greater impact through cross-unit (horizontal) alignment and partnerships. To achieve this within the group itself, we encouraged each group member not only to share the intercultural work of his/her unit, but also to explore more deeply how the different units represented in the group define and interpret intercultural issues.

\section{Securing Administrative Support}

Our group came to realize that efforts that require more cross-unit alignment, which could transcend institutional siloes, need to be intentional, consistently nurtured, and evaluated. The group acknowledged that an institution-wide intercultural advancement agenda requires having an engine that drives the effort and gives it the fuel and power it needs to go the distance. It was 
important to the group to see their discussions as not just another set of meetings, but as an effort that would have true institutional impact. One way that we (as the administrative leaders) sought to demonstrate the importance of the group's work was to provide direct staff and financial support from our offices to facilitate the group's next set of tasks. This support symbolized our continued commitment to the effort as a valued, University-wide strategic initiative. It also helped the group members see that they would have access to the necessary staff support (e.g., staff to coordinate and schedule the meetings, to document the group's discussions, etc.) so that their valuable time would be spent on building the agenda. Securing this administrative support proved to be an important practical and symbolic mechanism that helped recommit members to the group in ways that helped move the agenda-setting work forward. Our efforts were also buoyed by the encouragement and support we received from the University's president and the senior vice president for system academic administration.

\section{Questioning Standardized Assessments}

Another issue that helped propel the group's work forward had to do with how to best assess individuals', the group's and the institution's intercultural competence. Some members of the group were very invested in using standardized instruments. They believed that these tools opened the door for conversations with colleagues and provided a means to engage previously unengaged members of our community in self-reflection. However, others were concerned that the use of these established assessment tools made the work seem simple, easily achievable, and led to "intercultural competence lite,"' because the assessments focused on knowing one's score from the assessment, rather than focusing on what one needed to do to advance his or her intercultural understanding in an ongoing way.

There were also discussions about the limitations of most instruments, suggesting that existing instruments lacked comprehensiveness because they tend to view intercultural issues from a limited set of perspectives. Most instruments do not take into account other important, relevant perspectives. While the group agreed that it was important to have metrics in place, we could not agree on the scale, scope, or nature of the metrics for intercultural competence that would be most appropriate to use on a University-wide basis. However, as the group migrated away from using the term "intercultural competence" to define its work, we agreed that it would be advantageous to have several metrics in play to capture the group's broad-based understanding of intercultural issues.

\section{Phase II: Infusing New Perspectives}

As the group's work and discussions continued, we arrived at a point where we began to engage in circular conversations, revisiting previous conversation without moving the agenda forward. We became concerned about where the group's work was headed and how to keep everyone invested and engaged in the effort. We brainstormed various ways to operationalize more strategically the group's work so that we could move our intercultural agenda building work forward.

During the second year of our group's work, there was an opportunity to engage a group of professionals at the University not involved with intercultural work to collect data and 
information that we could use to guide our discussions. We seized this opportunity to bring in an outside, neutral perspective that could help provide us some new ways of looking at intercultural issues. This group - participants in a program known as the Presidents Emerging Leaders (PEL) — is composed of mid-level professionals interested in growing their own leadership capacity. Their work is actualized by group work in which they are engaged in new initiatives on campus. We engaged and worked with one PEL group to help us identify a set of recommendations for moving forward the University’s agenda for intercultural competence.

The PEL team worked on a relatively short time table and was charged with two tasks: (1) to identify contributions that the University can make to support development of individual's intercultural competence; and (2) to assess prevailing attitudes across the University community on diversity, engagement and internationalization as they pertain to the development of intercultural competence. The PEL team began benchmarking research at peer institutions focused on developing a campus-wide intercultural agenda. After significant effort, they were unsuccessful in finding another higher education institution in the United States that was immersed in this agenda-setting work. Surprised by this finding, the PEL team continued to look for examples at other institutions from which we might learn. But in the end, they were unable to identify any.

The PEL team then designed and administered a survey on the largest of our five campuses to assess the participating community members' understanding of and involvement with intercultural issues. This survey served as the basis for the PEL team's analysis and recommendations report to our working group. Of the 758 professionals identified as participating in intercultural work, 43 percent $(n=317)$ responded to the survey. When asked to describe the nature of their intercultural work, $74 \%$ of respondents to this survey item reported being directly engaged in some type of diversity-related work (See Table 2).

Table 2. Nature of Respondents' Work

\begin{tabular}{|c|c|}
\hline Nature of Work & $n=265$ \\
\hline Research, teaching, learning about diversity \& engaging the community & $31 \%$ \\
\hline Disability and diversity issues & $22 \%$ \\
\hline $\begin{array}{l}\text { Communication of information and educational opportunities in both } \\
\text { international and diverse environments }\end{array}$ & $21 \%$ \\
\hline Community engagement regarding health, social, and human issues. & $14 \%$ \\
\hline Outreach to external communities & $12 \%$ \\
\hline & $100 \%$ \\
\hline
\end{tabular}

Responses to other questions on the survey revealed that the primary focus and goals of intercultural work varied substantially among respondents. For example, one quarter of the respondents said that the primary goal of their work is to advance internationalization and address globalization issues; their approach to intercultural work is informed and shaped by national, ethic, language contexts. In contrast, another quarter of the respondents reported that they see their diversity work focused on bridging gaps by building bridges between groups and 
units from different parts of the campus; these respondents acknowledged that there are multiple perspectives to intercultural understanding, and that their approach is intentionally focused on helping units understand the different perspectives and lenses through which they conduct intercultural work.

In sum, the survey findings revealed that, while many individuals at the University are immersed in intercultural-related work, their views toward intercultural understanding is influenced in large part by the perspective of the unit with which they are affiliated. When considering respondents' primary unit affiliation (public engagement, equity and diversity, or international programs), their perspectives on intercultural understanding were informed by and generally aligned with their respective unit's focus. For example, respondents working with the Office for Public Engagement tend to have a community-empowerment perspective on intercultural understanding while respondents working with the Office for Equity and Diversity have more of a social justice and equity focus. Those who work with the Global Programs and Strategies Alliance office (international programs) tend to have a more nationally situated cultural view of intercultural understanding.

\section{Phase III: Accepting the Tensions}

Four significant tensions emerged from our discussions, cross-unit education, and survey analysis.

Inclusion vs. Cultural Appropriation

In our attempt to be an inclusive and understanding group, we inadvertently found ourselves engaged in what we term here as "offensive cultural appropriation”. Cultural appropriation is the adoption of some specific elements of one culture by a different cultural group, when not engaged in shared cultural exchange (Rogers, 2006). It can manifest in dress, artistic representations, language, or social behavior. Individuals in our group sought to appropriate into their work the others' definitions or perspectives about intercultural understanding to honor different points of views. However, because the definitions or perspectives are highly situational

— that is, their meaning are shaped by the nuanced contexts in which they occur - transporting them to new community or cultural contexts can result in establishing new meanings that are significantly divergent from those originally held. For example, the application of the construct of social justice (e.g., securing the fair and proper administration of laws and the equal treatment without prejudice) in one setting can be viewed in another setting as a perpetuation of injustice. Equal treatment does not equate to equity of outcomes; some might need something more, or something different, to achieve equitable outcomes. While some might see the provision of different levels or kinds of treatments to achieve equitable outcomes as a form of social justice, others might see this as socially and ethically unjust. Acknowledging and accepting the fact that inter-culturalism will manifest differently across different contexts, and with those with different histories and world views, is essential to building a university-wide agenda for intercultural understanding. 
Our group struggled mightily to determine where to draw the lines around what we identified as "culture". As a term embedded in the word "intercultural", we sought to unpack what was meant by "culture”. Is culture defined by groups and communities (e.g., by race, ethnicity, gender, gender expression or identity, sexual orientation, ways of thinking)? Or is culture an individual construct that is defined by one's own interpretation? Similarly, is it acceptable to have each member of the group (and the University) have his/her own definition of culture, or should there be a campus-wide set of understandings as to what we mean by culture? These were some of the questions that came to the fore for which we found no answers. Ultimately, we acknowledged and accepted the fact that the concept would remain amorphous and that we would focus on issues concerning both the individual and the group.

Connecting Identity to Inter-Culturalism

As we unpacked the issues of culture and subculture(s), our group realized that identities held within a subculture, particularly those that are marginalized, are key to understanding issues of culture. However, our group realized that issues of identity are generally absent from much of the literature on intercultural development, perhaps glossed over for the sake of making intercultural competence and development issues more simple and understandable. For example, issues of identity were not addressed adequately in most of the assessment instruments we discussed that measure intercultural competence and/or development. Yet, when engaged in various conversations about intercultural issues, whether it was through the lens of public engagement, equity and diversity, or international activities, the discussion of identity was prominent and necessary. This suggested to our group that more needs to be done in the field to link issues of identity to the intercultural advancement agenda.

\section{Difference vs. Commonality}

One of the main tensions in our discussions was how to best build commonality of understanding and practice across our cultures as we seek to build an institution-wide agenda for intercultural advancement. How much does focus on building a common agenda enhance or challenge our process? Does building a common agenda impede our ability to engage in critical thinking skills and to compare, understand, and appreciate the different values held across different cultures? Does developing a common agenda perpetuate a form of assimilation that preferences a dominant perspective or set of values? These were just some of the questions that emerged as our work on intercultural advancement began to take shape. We acknowledged that these kinds of questions would continue to be central to our discussions and would surely engage us in deep and difficult conversations.

\section{Phase IV: Refreshing the Agenda}

The external input and feedback we received from the PEL team clarified for us the importance of bringing a new, fresh perspective to our group's work. Such a perspective ultimately stretched our individual and collective thinking about what an institution-wide agenda for advancing intercultural understanding should encompass. To reflect this new phase of work, the group 
changed its name from the Intercultural Competence Work Group to the Collaborative for Intercultural Advancement (or CIA). This new name reflected the realization that building intercultural "competence" is just one piece of a multifaceted matrix of issues that need to be addressed if our institution is to build a comprehensive intercultural agenda.

With this new name came a new mission to reflect the shared goal of “advancement". The new mission statement became:

Building a university that fosters the capacity for students, staff, and faculty to work intentionally across cultures and contexts in creating inclusive, equitable, and reciprocal community and university partnerships for advancing the institution's mission.

The work of the CIA thereafter focused on achieving this mission through specific attention to addressing issues in four areas: faculty issues; staff issues; student issues; and communication

and assessment. Subgroups were created to work through the particular leverage points in each area to further the group’s new mission.

In articulating the next steps for the CIA, the members agreed that the advancement of intercultural understanding is not a time limited endeavor. Rather, it is developmental in nature and needs to be considered and cultivated on an ongoing basis. Also important was the acknowledgement that the work needs to go beyond western and global northern views, and incorporate perspectives from multiple cultures, nations, and regions of the world. The members also acknowledged the important role that language plays in the development of the agenda. Certain terms (e.g., social justice) may be interpreted very differently by different stakeholders, depending on the nature of the stakeholders' work and their frames of reference. Lastly, the group asserted that issues of intercultural advancement must be approached with an intersectional lens, and encompass all salient identity issues as well as the nuances for those with multiple identities.

With this new mission in mind, the group's agenda is poised take our University's intercultural work to the next level. Although the work of the CIA remains unfinished and continues, many lessons have been learned about what it takes to build an institution-wide agenda focused on advancing intercultural understanding. We hope that these lessons can help other higher education institutions interested in developing a campus-wide agenda for advancing intercultural understanding prepare themselves for the initial discovery, unpacking phases of the work, and addressing issues that arise.

\section{Key Lessons Learned}

During each phase of our work, we learned many important lessons. As more higher education institutions seek to advance intercultural understanding at their institutions, the following considerations may prove helpful in furthering their efforts. 
The Importance of a Campus-Wide Initiative

As we have described, we learned that there is wide-range of intercultural development work underway throughout our University. Yet, despite these various independent efforts, the institution lacked a campus-wide focus for advancing intercultural understanding. This supports the belief that broad-based intercultural advancement among faculty, staff, and students does not happen automatically (Deardorff, 2009). Rather, it requires an intentional effort and attention, through the establishment of a robust, campus-wide initiative, which brings together the various intercultural efforts by engaging key stakeholders in unpacking the various conceptualizations of the inter-culturalism, and organizing the conceptualizations into a cohesive institutional plan. Institutions engaged in such efforts should pay special attention to bringing together individuals and groups who have diverse perspectives and/or who do not necessarily agree on intercultural issues. Acknowledging that there is a broad range of perspectives, and ensuring that all perspectives are considered in the development of the institutional plan, is key to building a strong institution-wide plan to advance intercultural understanding.

\section{Establish a Trusting and Safe Space}

For the intercultural agenda to be successful, it is important that a safe space is established in which all stakeholders feel free to express their views no matter how much those views might differ from others. This space must be one that focuses on listening and understanding. It must embrace difference and dissonance. Creating this space was one of the key factors that allowed our group's work to advance because it helped engender a culture of trust that genuinely values every members' voice and participation. We also came to realize that creating this safe space actually required the group to practice intercultural understanding. Some individuals can find it quite difficult to speak openly and freely about sensitive issues in front of their professional peers, especially when senior administrators or other supervisors are present. Having a space for individuals to express themselves without judgment or criticism becomes key to be being able to do the deep dive that is necessary to unpack the complexities of intercultural understanding. One the important lessons learned in this regard is to not avoid discussions about different perspectives, contested language, or difficult issues and to take the time necessary for all to feel heard and to learn from one another. In our group's work, differences of opinion were valued and seen as opportunities to educate the group and to garner greater understanding of the complex character and core of intercultural understanding. To this end, institutions that form work groups to build an institution-wide intercultural understanding agenda should consider the extent to which the group itself models the best practices that it is attempting to advance.

\section{Honor existing work}

Given that most of the participants in our group had had extensive experience with intercultural issues, it was important to honor the existing experience, expertise, knowledge, and understanding that the individuals and their units brought to the table. The goal of developing a campus-wide agenda was not meant to supplant existing work, by establishing an intercultural agenda that focused on a specific set of principles or practices. Rather the goal was to take stock of the wide range of existing work, assess the differences and similarities in approaches, acknowledge the contribution of each effort, and develop a comprehensive agenda that embraces 
all of the work, in ways that leverage the institutional impact to deepen understanding across the institution.

\section{Bring in Outside Perspectives}

The use of the PEL team helped our group to provide a mission check and feedback about our group's work. It is not difficult for such work to become biased as the perspectives of those with the loudest or most influential voices sway group members' opinions and viewpoints. Bringing in a team that provides a neutral perspective can prove beneficial to keeping the group grounded in the established mission, and if necessary, help align the group's work to its original intended goals. This external team can also provide data and evidence about the group's process and offer an honest assessment of the group's successes and limitations. Such feedback is a launching pad for infusing new ideas into the group’s work.

View Intercultural Understanding as a Strategy, Not a Goal

At one point in our group's work, we began to lose sight of why we were engaged in the effort. We were so immersed in trying to understand each other's perspectives regarding intercultural understanding that our goal had turned to defining intercultural competence, development, and understanding. However, we arrived at a point where we started to question why we were doing this work and why establishing a campus-wide agenda for intercultural understanding was important. Through this reflection, we reaffirmed and re-anchored our group's focus to our initial goal: creating an institutional agenda for intercultural understanding for the purpose of strengthening the quality of relationships that members of the University held with each other and with various external partners and communities. If we achieved these stronger and deeper relationships, our University would be able to enhance individuals' and groups' sense of belonging, secure a more inclusive and accepting institutional climate, and further the impact of the University's community engagement, equity and diversity and international programs efforts. In sum, keeping these broader goals front and center is essential for maintaining the initiative's institutional value and for realizing the initiative's contribution to advancing the institution's overarching mission.

\section{Acknowledge the Limitations of Campus-wide Efforts}

Despite senior leaders' support for developing the agenda, and despite the participation of committed and knowledgeable campus stakeholders, our experience with the work group suggests that the real leverage for change takes place within the individual units for whom intercultural work is primary and central. As our effort revealed, the operationalization of intercultural work is widely distributed and situational. While a campus-wide (or institutionwide) effort can provide guidance and expectations, it is less likely to be able to set influential institutional policy or to affect actual practice. Such impact will likely depend on the extent to which the individual programs legitimize and promote intercultural advancement within the work of their units. In the end, the leverage for real change does not reside at the campus level, but back within individual administrative and academic programs and the individuals within these programs who operationalize intercultural practices every day. 


\section{Give it time}

Lastly, perhaps the greatest lesson of all, is that establishing and cultivating a robust institutionwide agenda for advancing intercultural understanding takes a great deal of time. Since the initiative is about changing the institutional culture, it requires staying the course over time and throughout changes in leadership and personnel. It requires digging deeply into complex and sensitive issues, and the work necessarily will expose many of the institution's shortcomings and limitations. The discussions and process are often difficult and challenging. In the end, however, it is being able to stay the course to work through these challenges that will bring the institution to a new level of intercultural understanding.

\section{Looking to the Future}

The effort we undertook has catalyzed additional conversations about the importance of understanding the different frames and perspectives that undergird intercultural understanding. Specifically, we have seen the implementation of a series of forums that are engaging different stakeholder groups, both within and outside of the University, in critical conversations about a variety of intercultural issues (i.e., race, ethnicity, gender, sexual orientation, class, language). These conversations have taken the form of scholarly presentations, debates, roundtable discussions, invited speakers, artist renderings, and theatrical and dance productions. Examining the issues through multiple forums and modalities has proven effective in attracting a broader range of stakeholders to the conversation. These critical conversations appear to have also engendered a greater comfortability among some who have previously found it difficult to hold frank discussions on intercultural-related issues.

The increased polarizing rhetoric we are finding within our society has made it even more crucial for our higher education institutions to provide much needed spaces for groups and individuals to reflect on their intercultural understanding, attitudes, and behaviors. Moreover, with the discourse and debates becoming increasingly political, there is an urgent need for higher education institutions to provide education, training, and skills development on how to build and strengthen one's intercultural competencies and understanding. As we had learned from our work, such competencies and understanding do not manifest automatically. Intentional, concerted, and strategic efforts that engage faculty, staff, students, and key administrative units are required in order to secure an institutional culture that genuinely embraces and values interculturalism. As Deardorff (2009) reminds us, building such a culture within higher education institutions takes strategic commitment and time. As our University's commitment to this important work persists, we continue to search for the nodes of opportunity that we can leverage to further the intercultural advancement agenda.

\section{Conclusion}

We realize that we were, and are, engaged in work that needs to be done slowly, patiently, and with the understanding that the how is as important as the why and the what. And while our goal to work with University partners engaged in fostering intercultural awareness and understanding to support the development of competency in others, our work to date has in large part involved deepening our own intercultural awareness across our own different fields and philosophical 
approaches to the work. It is our hope that in sharing the story of our journey thus far, others will be encouraged to take on the challenge of establishing bold agendas for advancing intercultural understanding at their institutions, and secure on their campuses a foundation for deep intercultural understanding that enhances each institution's mission and maximizes the potential for the success of all members of the community. 


\section{References}

Deardorff, D.K. (Ed.) (2009). The Sage handbook of intercultural competence. Thousand Oaks: Sage Publications.

Rogers, R. A. (2006). From cultural exchange to transculturation: A review and reconceptualization of cultural appropriation. Communication Theory, 16(4), 474-503. https://doi.org/10.1111/j.1468-2885.2006.00277.x 


\section{Author Information}

Andrew Furco is Associate Vice President for Public Engagement at the University of

Minnesota, where he is also a professor of higher education in College of Education and Human Development. His research focuses on studying and assessing the role of community engagement in educational systems (primary, secondary, and higher education) in the United States and abroad. Prior to arriving in Minnesota, he served for 14 years as the founding director of the Service-Learning Research and Development Center at the University of California, Berkeley.

* Andrew Furco

Office for Public Engagement

University of Minnesota

110 Morrill Hall

100 Church Street, S.E.

Minneapolis, MN 55455

Email: afurco@umn.edu

Telephone: 612-624-6876

Kristin Nering Lockhart was the Associate Vice President for Equity and Diversity at the University of Minnesota and worked on social justice issues related to diversity, inclusion, equal opportunity and affirmative action for over 30 years in the legal field, with corporations and nonprofits, and in educational arenas. Kris was an administrator working on equity and diversity issues at the University for over 18 years.

Kris Lockhart

Email: lockhart@umn.edu

*Corresponding author 